\title{
Social Justice as an Organizational Identity: An Inductive Case Study Examining the Role of Diversity and Inclusivity Initiatives in Corporate Climate and Productivity
}

\author{
Kathryn Thomas \\ Yale University \\ Chase Ochrach \\ University of Wisconsin-Madison \\ Brian Phillips \\ Utah State University \\ Timothy Tansey \\ University of Wisconsin-Madison
}

Although research has begun to establish the benefits of implementing disability inclusion initiatives within companies, there is relatively limited research examining the impact of applied diversity management practices in the employment of PWDs. This study explores the disability initiatives of an exemplar midwestern company through an in-depth case study. An inductive, qualitative design was utilized to identify themes from interviews with multiple employees. Interviews were examined to understand how the company's social mission and corporate identity impacted productivity and perceptions. Results suggest that employees are drawn to the company by their social justice mission in hiring and creating meaningful employment for persons with disabilities. Moreover, the fact that the company sees this mission as a central part of its organizational identity rather than as an ancillary initiative motivates employees. Findings indicate that centering a commitment to hire and support persons with disabilities within the organizational identity, forming partnerships within the community, and connecting employees with a sense of meaning in their work generates greater productivity, engagement, and commitment from employees.

Keywords: diversity, inclusivity, corporate climate, productivity, social justice, diversity initiative, company culture

\section{INTRODUCTION}

People with disabilities (PWDs) compose approximately 26 percent of the potential workforce in the United States (Centers for Disease Control and Prevention, 2018) but are drastically underrepresented in the employment rates. Only 17.9 percent of PWDs were employed in 2020 compared to 61.8 percent for those without disabilities (U.S. Department of Labor Bureau of Labor Statistics, 2021). To address the need 
for employers to find qualified workers and for people with disabilities to contribute to the workplace, a body of literature has emerged examining the hiring and retention of employees with disabilities and how it benefits the company, the employees with disabilities, and their co-workers. While prior research emphasized the negative impacts of unemployment on the wellbeing of PWDs, recent research has demonstrated the benefits of employing PWDs to companies. A recent meta-analysis revealed that employing PWDs benefitted companies in several ways, including increased profitability, a competitive advantage in recruiting qualified employees, a more inclusive work culture, and greater ability awareness (Lindsay et al., 2018). Specifically, in terms of profitability, results revealed that several companies reported improved profits from hiring PWDs. These increased profits were attributed to increased sales, cost savings of not having to rehire and retrain new employees, higher rates of attendance and reliability of PWDs, improved loyalty of employees, expanded customer base, improved customer loyalty and satisfaction, and tax benefits and incentives (Lindsay et al., 2018). Research has also revealed the benefits of hiring PWDs for the workplace culture overall, such that the diversification of work settings leads to a more positive, inclusive work environment and improved morale for all employees (Lindsay et al., 2018).

Of course, the gains from hiring PWDs are not automatic. Poor process and inaccurate perceptions can limit potential gains. The Diversity Identity Management Model provides a useful framework for understanding how companies can facilitate the employment of PWDs (Cole \& Salimath, 2013). The Diversity Identity Management Model is based in the Institutional Theory (Selznick, 1949; DiMaggio \& Powell, 1983), which provides an understanding of how structures, such as rules, norms, and other criteria of social behavior, are formed by society and become ingrained in companies. The Diversity Identity Management Model includes three key propositions that explain how diversity identity can be incorporated into a company from both a top-down and bottom-up approach. First, the model posits that stakeholder expectations for diversity create institutional pressure that affects the external legitimacy and diversity identity of companies (Cole \& Salimath, 2013). Stakeholder expectations include coercive pressures (a threat of adverse consequences if the organization does not conform with external laws and expectations), normative pressures (unspoken pressures and expectations communicated through employees of the organization), and mimetic effects (pressures to promote similar formal structures to other organizations and companies in the same field; Cole \& Salimath, 2013).

Second, proactive responses to stakeholder pressure for diversity lead to the formal incorporation of diversity into a company's identity (Cole \& Salimath, 2013). Organizational identity is dynamic, thus allowing for organizations to respond to institutional pressures to promote diversity by proactively incorporating diversity into their identity. Third, an optimal level of incorporation of diversity into a company's identity creates positive organizational diversity identity and minimizes diversity identity threats (Cole \& Salimath, 2013). The theory posits that inadequate or excessive diversity identity management (i.e., over or underemphasizing diversity) can lead to resistance, discontent, and threats to an organization's diversity identity (Cole \& Salimath, 2013). In summary, the Diversity Identity Management Model provides a theoretical model for understanding proactive responses to diversity issues, such that stakeholder pressures to promote diversity leads to the incorporation of diversity identity; with the management of the company's diversity identity, these efforts can lead to a perception of legitimacy and diversity identity (Cole \& Salimath, 2013).

Although research has begun to establish the benefits of implementing disability inclusion initiatives (e.g., Hindle et al., 2010; Kalargyrou, 2014; Lindsay et al., 2018), there is relatively limited research examining the impact of applied diversity management practices in the employment of PWDs. Cavanagh et al. (2017) concluded that there is a dearth of literature on the management of people with disabilities at the workplace. Therefore, the purpose of this study is to explore disability initiatives and diversity management practices in a midwestern software company, Yolobe. By applying the Diversity Identity Management Model to our understanding of Yolobe's practices of hiring, supporting, and retaining PWDs, the study aims to provide a framework for how other companies can implement diversity management practices and promote the hiring of PWDs to the benefit of the company, PWDs, and employees more generally. 
Yolobe is a software company located in Chicago, Illinois, with a social mission to "dramatically improve the lives of young adults and working-age adults with disabilities by connecting them to services and opportunities, jobs, training, and supportive services in order to be successful." Yolobe provides a software platform that facilitates professional social networking for people with disabilities by creating a place to network and to find and share internships, mentoring, and jobs. Yolobe employed approximately 20 people at the time this research was conducted.

\section{METHODS}

We implemented an in-depth case study of a company that has an expressed commitment to inclusive hiring and retention of PWDs based on a clear recognition of the company-wide benefits of hiring PWDs. The goal of the case study was to gain a deeper understanding of how the company's missions and values translate to policy and practice of hiring and retaining employees with disabilities. This study was guided by the following questions: (a) What are the overarching attitudes, values, and approaches taken by this company in their efforts to be inclusive and diverse? And, (b) How does this company extend its message of wellbeing and support throughout all levels and departments of the company?

Given the formative nature of this investigation, a qualitative case study design was used to allow for the exploration of events or phenomena from multiple sources to explore the unique experience of participants' lives and interactions with the environment (Corbin \& Strauss, 2015). Prior research has suggested that qualitative case studies on organizational best practices are an ideal approach to unpacking the impact of effective practices and supportive management in the employment of PWDs (Cavanagh et al., 2017). This inductive qualitative design allowed for an open evaluation of the complexities and considerations involved in recruiting, hiring, supporting, and retaining PWDs through in-depth interviews with multiple staff members at different levels within the company (Maxwell, 1996). Due to the exploratory nature of the study and our goal of amplifying the voices of the participants at all levels of the company, a qualitative approach was necessary (Miles \& Huberman, 1994). The research team involved in data collection and analysis consisted of six rehabilitation psychology, rehabilitation counseling, and counseling psychology faculty and doctoral students across three universities in the United States. Team members discussed potential biases prior to engaging in data collection and again before completing the analysis to reduce the potential for biases to influence the results.

\section{RESULTS}

This case study focuses on the attitudinal and cultural approach one company has taken to prioritizing diversity - specifically by valuing PWDs - in the workplace. This company connects young adults with a variety of ability backgrounds who are searching for jobs with companies outwardly invested in hiring a diverse workforce. This case study focuses on the overarching mission, core values, and lived experience of this company's orientation towards placing PWDs in skill-based positions through community partnerships. The results of this investigation were drawn from interviews with the CEO of the company, employees across a range of positions within the company, and the internship coordinator. The results of this study are broken down into major themes and findings related to (a) the CEO's values, (b) company mission, (c) factors that contributed to the company's success, and (d) thoughts about how to replicate this success.

\section{Grounded in Transparency, Empathy, and Vulnerability: CEO's Reflections}

The company CEO clearly articulated the three main values in which the company is grounded: transparency, vulnerability, and empathy. The CEO expanded first on transparency, describing how he hired an outside consultant to encourage a culture of transparency and vulnerability in the company:

I'm trying to build a culture of transparency, yes, but I'm trying to build a culture where vulnerability is considered a strength. As part of that effort, we've actually hired an external 
coach to come work with my team, all of my team, on a number of things, including the willingness to be vulnerable.

He continued,

One of the things we're teaching our people is that it's okay to divulge things in a safe environment to the people that you trust, and there's strength in that because it allows for empathy, growth, and connection to happen. And it builds a team and a company that's more resilient.

The CEO explained that through transparency and vulnerability, his employees were able to understand, connect with, and have empathy for one another. He stated, "My team is much more empathetic, and I've actually learned that there are disabilities within my team that I had not been aware of, but now that I am, I'm very happy about [it]. It's given me a renewed sense of empathy towards them and connection."

\section{Mission}

The company CEO described the company as, "A software company [that] has a social mission, which is to dramatically improve the lives of young adults, teenagers, and working-age adults with disabilities primarily by connecting them to the services and the opportunities (jobs, training, supportive services, and so on), to be successful."

He described the company as a three-sided marketplace that includes (a) PWDs seeking opportunities, (b) the software application that can be operated by partner organizations, and (c) organizations providing opportunities or offering supportive services to build connections between employers and job seekers. The CEO stated that this model, "Allows third-party organizations, such as employers, to knowingly come into a community that's serving the disability community, access information related to that community, as well as benefit from the relationships of the organizations serving [PWDs]."

Furthermore, the CEO referred frequently to the company's mission and values that center on diversity, stating,

In our mission statement, access to opportunity and equality of opportunity for those with disabilities is something that we're striving for. By virtue of [these values], we are incredibly open to having a set of employees who are diverse. And so, we actively seek that out and see that as a strength. That's embedded in our mission statement.

Part of that mission comes down to an equal emphasis on being both a socially conscious and solutionfocused company. As a firm geared towards making connections and finding solutions for clients, this company has turned its marketable services inwards, searching for solutions for its employees in-house. The CEO stated,

We built a company with very much a social mission and primarily targeting underserved populations who have traditionally been overlooked by the workplace in general. I've made it a mission of mine to try to come up with solutions to help in that particular space.

Speaking more to the solutions this company has generated for its employees, he continued,

I tirelessly pursued working and trying to build solutions for people with disabilities. It's all been centered around the question of how to drive more employment to [PWDs] and put them on a path where they are able to take care of themselves and hopefully their families in a manner that gives them high dignity. 


\section{Diversity and Inclusivity}

Part of the company's lived mission is apparent in its focus on diversity and inclusivity. Not only is the company designed to match job-seekers with disabilities with well-suited employment opportunities with their community partners, but they also offer flexibility to all employees to be able to work from geographically diverse locations. In a focus-group interview with current employees of the company, individuals called in from three different countries. These employees shared how they valued having the flexibility to work from home and that the company accepted the challenges that can come from employees being in different time zones.

The company seeks to promote inclusivity through its platform by making it accessible for others to use. As the chief technology official explained,

As we build this platform, we are looking into all those aspects of effectiveness, not just in terms of our application being accessible, but how do we make sure that our networks, like Equal Access and [PWDs] who are using our platform, are actually getting benefit from this platform to find their roles, find their jobs and be in a position to make this platform useable.

This respondent further explained that, as the platform evolves and new features are added, the company utilizes a web-based tool to determine how accessible the platform is for individuals with different ability levels.

Beyond the platform itself, the company recognizes the benefit of bringing PWDs into the problemsolving process throughout the company. As the CEO shared,

If you include [employees with disabilities] in identifying gaps and then in the development of the solution to [those gaps], and that is regarded as a priority item for the employer to address, then you build a solid company that is very inclusive.

One product of this collaborative problem-solving has been for the company to include certain keywords in their posts about different job opportunities that highlight the requirements, skills, or potential accommodations PWDs would benefit from knowing about before applying to the job.

One additional piece in promoting inclusivity from the workplace comes from community partners and organizations that are all invested in the same goal. As the internship coordinator shared,

I would say our biggest effort was our group of organizations that are part of our Equal Access network, which is about seven or eight organizations around the Chicago area that are specifically focused on providing jobs and opportunities to [PWDs]. Our goal there is to not just be a platform that provides any type of job, but really getting companies and employers on board with our platform where we are providing those opportunities and specifically looking for people with disabilities with the goal to diversify their workforce.

All of these factors - searching for diversified talent, flexible work environments and scheduling, and community partnerships with the same goals and values - are foundational to the company's success.

\section{Importance of Community}

The CEO underscored the importance of community both within the company and between the company and the organizations they partner with. This sense of community helps drive the corporate mission and values throughout the company and allows for seamless exchanges between the company, jobseekers, and employers. The CEO expanded on how this makes their company different,

Historically, there's a lot of silos. Organizations have been independently working to build relationships with employers, trying to satisfy questions employers might have about hiring 
somebody with a disability, trying to independently share success stories, and get employers interested and excited about hiring somebody with a disability. We believe that the right approach to solving [employment rates of people with disabilities] at this scale is to approach this from a community standpoint, both in terms of the technology that's being applied to it as well as the messaging so that it's a common framework that all of the participants are using and a common set of practices if they're participating.

The CEO further described how the employers who work with the company are intentional about their decision to hire PWDs and value them for their strengths. He explained how the partnerships and systems the company put in place offers structure and support for both the PWD employees and their employers as follows:

The employers look at the hiring of somebody with disabilities, not as a rote, you know, "look at this person's résumé and profile and make a decision," which is typically what would be done today on any existing platform, but rather a partnership with the career specialist who knows these people who are applying and therefore can give them the social capital that has been missing. For many in the entry-level space, that is what they need to qualify for an opportunity or be given the opportunity to potentially participate in [the labor market].

The CEO explained how the felt sense of community within the company allowed employees to be honest and authentic about their skills and disability statuses, stating,

There's a benefit to having people self-identify with having a disability as opposed to hiding it, which is currently what's oftentimes happening today. Our approach is very much about having people with disabilities opt into this understanding that they seek a community that's supportive of them.

\section{Impacts of Mission}

Across all interviews at all levels, participants spoke to the unique impacts of the company's goals and mission on their perception of and experience with the company as well as on the company's overall climate.

\section{Impact of Mission on Employees}

Having a sense of meaning in the workplace came up in all employee interviews. As one member of the engineering team described,

If I don't feel like I have a mission, I normally stop being productive. What I mean is not completely obvious, but when I see my levels of productivity drop it means that I need a change. And that actually answers your question, that at Yolobe I feel really productive. We are a small team, but we are delivering big things already. Even being small, we are already productive and developing big things, so I would say it impacts a lot.

The internship coordinator shared how working with PWDs and operating in service of the company's mission has inspired her growth and learning, stating

I learned a lot because...I never worked with a group of people who learned in that way, and so the way that I had to go about telling them things or teaching them was a lot different. I learned a lot personally on how to structure what I was saying or what I was doing so that they could understand better, but it wasn't necessarily something that was 
negative. I feel like everything that I learned from working with [PWDs] was either neutral or I was changed for the better.

Several employees described that interacting with PWDs through the company has served as a motivating force. As another member of the engineering team shared,

The most active and most interesting channels on Yolobe are all related to the [PWDs]. I interact with general administrators more often, so we definitely get some specific requests addressing the needs of [PWDs]. It keeps us motivated and it keeps us innovative. For example, recently we are making a big change to our platform and it's all about addressing those specific needs in terms of the [PWDs'] profiles on the system.

The head of marketing reflected on the importance of feedback between the company developing the platform and the PWDs using it, explaining "As part of my job, I get to hear what [employees with disabilities] want, what they're going through, how we can work with them better. It motivates me knowing our users and getting to know who we're working with."

Working somewhere with an overall sense of mission, connecting with individuals who benefit from the company, and using their feedback as motivation all seem to create a satisfying workplace for employees at the company. The CEO noted a keen awareness of the impact of a sense of meaning on employment, stating

I'm always looking to make sure that people feel like they're doing valuable work. I can connect them on a continuous basis to how the work that they're doing is, in fact, valuable and that has helped to build a very cohesive team structure for us. And then, outside of that, encouraging and nurturing their involvement in any other groups.

\section{Impact of Mission on Company Culture}

Beyond individual impacts, the company mission has affected the overall culture and climate of the company as a whole. A member of the engineering team spoke to the cohesion he experiences at the company,

The fact that we are all working together to build something that will make a difference to a lot of people who normally get forgotten, brings a sense of unity. I'm not sure how I can express this in words, but if we are all embracing the same mission, it actually pulls us together. It makes our efforts and our well-being as a team, our chemistry as a team, work better, so I would say that the impact has always been positive so far.

The culture is certainly a force in the retention of all employees, especially those that find value in the work of the company. The CEO shared his belief that retention rates of employees are high when the employees perceive their work as "valuable" and themselves as "valued" by the company.

\section{Factors of Success}

Training

The CEO shared that a large part of the company's success was due to their focus on the training and onboarding process for all employees. He explained how the companies who see the greatest return on investment from the people they hire are those that emphasize training and keeping employees current on company goals and workflow. He stated,

Hiring somebody is the easy part of the job, assuming that they're going to fill the niche that they've been hired for without further work is an oversight that most managers make. The key role of a good manager in bringing somebody on board is to recognize that, once 
they're on board, that's when the job of being a manager truly starts, and that's the hard part.

The CEO went on to say that training is most effective within the context of a healthy relationship between employer and employee. He states,

What needs to happen is relationship building, and this is where it is critical to have a good culture where transparency, being empathetic, and being able to have the types of conversations that a new employee would need to feel like they are being best served can happen. What you really need is an environment where somebody feels like they're set up to succeed. And, dependent upon the person, it's always going to be different.

\section{Meaning}

Related to being intentional with the training and onboarding process, as well as building a relationship between the employee and their new workplace community, a continuing theme was the importance of having a sense of purpose. Speaking to current and potential employees' perception of the company, the CEO stated,

[Our social mission] has been a strong driving factor. In fact, it's one of the big common denominators of things the people that I'm interviewing are after. Not only do they enjoy the work they're doing, but they're very happy that the work they're doing has the potential to help hundreds if not millions of people.

The employees reiterated that they are inspired by the company's social mission. The head of marketing shared her decision to join the company was inspired by her background in social policy and her being motivated to "help people." Other employees connected to this message as well, as one member of the engineering team shared the pride derived from the company's mission by saying,

I joined [the company] with this feeling since the very beginning, and as our journey goes along I feel even more immersed in our mission. I'm really happy to be here. You do feel more realized when you feel you are making a difference; when you actually look at what you're doing every day and you feel happy.

\section{Partnerships}

The CEO identifies the employers on the company's platform as the "early adopters," who, because the company is only in its early stages, must "genuinely have a particular passion and/or interest in hiring somebody with a disability." Speaking of their partners more generally, the CEO explained the variety of reasons the company has partners by saying,

[Some] organizations actively employ people with disabilities specifically because they have a particular skill set. For example, using people with autism to conduct softwarebased testing. Clearly, there is a specific need for them to be recruited to that space. And then there are other organizations that are run by people genuinely passionate about adding to the diversity that they currently have in-house.

The CEO also acknowledged that some potential partners may be reluctant about hiring PWDs because they are unsure of how best to support them. To alleviate this resistance, he stated,

We believe that, as part of the solution that we're building, we need to not only provide access to people with disabilities and the people who work with them but also have a set of resources that they can tap into that reflect the current state of the art in thinking as well 
as the current state of the art in policies and procedures and so on. We believe that this will help alleviate some of the common patterns or thought processes employers have today that may have precluded them from going down the path of hiring somebody with a disability.

\section{Replication}

When asked how other companies might be able to replicate the success of Yolobe, the employees and CEO had several insights. The CEO's first recommendation was to embrace diversity, suggesting,

Having more diversity and, in particular, hiring more people with disabilities would benefit virtually every if not all companies. Most companies are not set up to do that effectively, but I don't think it's out of their reach. It really depends upon establishing the right sort of cultural framework for that to happen.

He further suggested capitalizing on community partnerships where the other company is interested in working with your company long-term. That strong relationship, he argued, can serve as a testament to other organizations who may be interested in forming a partnership. The CEO also noted that many organizations would be willing to form this kind of partnership, and emphasized the importance of explaining the benefit to the company, PWDs, and the community in pitching it. He stated,

Some portion of people will gravitate [to this type of partnership] and are more amenable because they have a higher empathy or have some personal experience. Given the right sets of tools, these companies can adopt effective practices quite easily because they put forth the investment. Then others will be harder pressed to do so but would be willing to do so if presented with the right arguments of how this will actually benefit their organization.

One pathway to engaging the community more broadly would be for whole cities to partner with organizations that have a built-in diversity initiative. The CEO explained,

Cities, for instance. There's a real potential here just because of the clout a city or a state has. Absent the fact that many of them are already working with big providers who have to be inclusive, part of what we're banking on is that organizations that are working with our cities are going to want to be inclusive because they've been asked by the city to be a part of this effort. I think it will make a lot more inroads, especially if we go in armed with a set of methods for companies that traditionally haven't been very compliant or traditionally haven't offered opportunities to people with disabilities.

One actionable step to bring diversity and inclusivity initiatives into the company is by starting a disabilityfocused internship program, as was done at Yolobe. The internship coordinator explained,

Having the internship is a great experience and opportunity for any company. Obviously we want all companies to be diverse, but if a company is not willing to do that or able to do that, having an internship is a great option because you gain a lot of knowledge and information and experience as a company, and so do the individuals who are part of the program.

Using an internship program model could be a way for a company to begin to become familiar with the unique skills and needs of employees with disabilities. Companies using an internship program could use it as an opportunity to learn and create the infrastructure needed for the long-term employment of PWDs. 


\section{DISCUSSION}

The following discussion examines Yolobe's employment of PWDs through the lens of the Diversity Identity Management Model (Cole \& Salimath, 2013), which provides an understanding of how structures, such as rules, norms, and other criteria of social behavior, are formed by society and become ingrained in companies. Specifically, the Diversity Identity Management Model is based on the following three propositions: (a) Stakeholder expectations of diversity create institutional pressure that affects external legitimacy and diversity identity of companies, (b) Proactive responses to stakeholder pressure for diversity lead to the formal incorporation of diversity into a company's identity, and (c) An optimal level of incorporation of diversity into a company's identity creates positive organizational diversity identity and minimizes diversity identity threat (Cole \& Salimath, 2013).

\section{Stakeholder Expectation of Diversity}

Yolobe manifests the first proposition of the Diversity Identity Management model, which suggests that stakeholder expectations of diversity create institutional pressure that affects external legitimacy and diversity identity of companies (Cole \& Salimath, 2013). Stakeholder expectations include coercive pressures (a threat of adverse consequences if the organization does not conform with external laws and expectations), normative pressures (unspoken pressures and expectations communicated through employees of the organization), and mimetic effects (pressures to promote similar formal structures to other organizations and companies in the same field; Cole \& Salimath, 2013). With laws, such as the Americans with Disabilities Act (ADA) of 1990, companies in the United States face the external pressure to value disability and conform with the legal requirements. One employee described this external pressure and contrasted it with a genuine desire for diversity by stating that some companies "genuinely want to provide accessibility because that's part of who they are, and they think it's the right thing to do. I totally agree with that." In contrast, other companies, the employee stated, are more "wary of being sued." It is important to note that these legal requirements were perceived, at times, as barriers to furthering the mission of employing PWDs, as in the following:

We are very interested in pursuing ways that employers can more regularly interface and interact in a less formal way with others to drive more opportunities to folks. How do you balance that against this litigious environment that we work in, which is, unfortunately, hampering many people who have a disability in terms of their ability to find employment? It's an unspoken truth that people are wary of potential lawsuits.

Beyond the legal requirements and expectations to conform with legal requirements, the CEO of Yolobe is a stakeholder who created a culture that legitimized the diversity identity of Yolobe through values that were incorporated on all levels of the company. The CEO described the three main values of Yolobe as transparency, vulnerability, and empathy, such that employees perceived a safe environment to share their authentic selves and provide empathy to one another. This empathic environment facilitated a stakeholder expectation of diversity, as there was a clear institutional expectation to respect one another's identities and values.

In addition to the company values, the mission of Yolobe furthered the stakeholder expectation of diversity. The $\mathrm{CEO}$ described the mission of Yolobe as,

A software company [that] has a social mission, which is to dramatically improve the lives of young adults, teens, and working-age adults with disabilities, primarily by connecting them to the services and the opportunities, jobs, training, supportive services to be successful.

By creating a mission with a clear purpose of employing PWDs, the CEO created a stakeholder expectation that diversity will be valued within all levels of the company. A focus group conducted with 
current employees of Yolobe confirmed their perception of the value of diversity within the company, as evidenced by the flexibility they were given in their work to best meet their individual needs.

\section{Proactive Responses to Stakeholder Pressures}

Yolobe further embodies the second proposition of the Diversity Identity Management Model, which suggests that when a company is proactive in its response to pressures from stakeholders within and outside of the company to implement diversity initiatives, these initiatives then become a part of the company's identity (Cole \& Salimath, 2013). Interview participants from Yolobe clearly identified with the diversity focus of the company and reported being attracted to the company initially from this mission and enjoying their sense of purpose at work driven by that mission. The internship coordinator shared,

I always knew that Yolobe had a great mission because they were a company that wanted to provide people with opportunities. But the fact that they wanted to become a company that was not only inclusive in their goal to connect other companies to people with disabilities or people with disabilities to opportunities, but they were also willing themselves to take on people with disabilities. It just put them up higher in my mind, because I already thought of them pretty highly.

Potential employees are drawn to the company because of their focus on inclusivity within their team and the large role that focus plays in their corporate identity. Cole and Salimath (2013) argue that companies that take on these initiatives without integrating them into the company's identity will face internal and external threats to the perception of the legitimacy of their diversity focus, which can deteriorate corporate unity and climate. This is consistent with findings from previous research that employees are more productive, experience greater job and life satisfaction, demonstrate greater work engagement and job performance, and are more committed to the company as a whole when they feel that their work is meaningful (Allan et al., 2019; Chalofsky, 2003; Yeoman, 2014). This sense of meaning is much more salient when employees perceive social justice as a part of their corporate community's identity rather than an individual identity among some employees. Across all participant interviews, Yolobe employees demonstrated a genuine integration of diversity and inclusion into their identity in recruiting, hiring, and supporting PWDs.

\section{Optimal Level of Incorporation of Diversity Identity}

Yolobe also embodies the third proposition of the Diversity Identity Management Model, which posits that an optimal level of incorporation of diversity into a company's identity creates positive organizational diversity identity and minimizes diversity identity threat (Cole \& Salimath, 2013). Further, the theory suggests that inadequate or excessive diversity identity management (i.e., over or underemphasizing diversity) can lead to resistance, discontent, and threats to an organization's diversity identity (Cole \& Salimath, 2013). Data collected as a part of the case study provides support that Yolobe has achieved an optimal level of incorporation of diversity into the company's identity, resulting in positive organization diversity identity and minimal diversity identity threat. By incorporating diversity, in particular the value of employing PWDs, into the values and missions of the company, the CEO created a company culture that values diversity and promotes empathy and acceptance of each employee's individual needs and abilities. The emphasis on diversity within Yolobe strikes an optimal level of diversity identity through value-driven leadership and flexibility that optimized the strengths and abilities of all employees.

\section{Limitations}

This case study utilized in-depth interviews with the CEO, the internship coordinator, and several employees in different positions and working from different geographic locations throughout the company. Findings from this study are meaningful and carry important implications for the integration of a social justice mission into the workplace identity and the role of meaning and purpose in employee recruitment and retention. However, there are important limitations in this study. First, this study examines Yolobe's 
efforts to recruit, hire, and support PWDs into their own organization and to help PWDs in the community find meaningful employment with their partner organizations. However, neither Yolobe employees who are PWDs nor Yolobe clients who are PWDs were interviewed for this study. Future research should examine PWDs accounts of their experiences working for companies with diversity initiatives. An additional limitation is the omission of interviews with community partners in this study. The importance of community partnerships repeatedly emerged across interviews for this study. Future research should incorporate the voices of members of these partner organizations to understand how companies effectively partner with organizations in the community to meet their productivity and social goals.

\section{CONCLUSION}

Yolobe is a company founded upon a social mission. The company founders recognized a social and industry need to help PWDs find meaningful employment. They also recognized the benefits to companies who hire PWDs and emphasized how the recruitment and hiring of PWDs was mutually beneficial to all stakeholders. The success of this company is grounded in their social justice focus, the infusion of meaning and purpose in the tasks of employees at all levels, and their sense of unity within the organization and with community partners. Our inductive case study approach revealed that a strong corporate mission that has become fully integrated as a part of the corporate identity is key to promoting buy-in from both employees and clients.

\section{REFERENCES}

Allan, B.A., Batz-Barbarich, C., Sterling, H.M., \& Tay, L. (2019). Outcomes of meaningful work: A meta-analysis. Journal of Management Studies, 56(3), 500-528.

Cavanagh, J., Bartram, T., Meacham, H., Bigby, C., Oakman, J., \& Fossey, E. (2017). Supporting workers with disabilities: A scoping review of the role of human resource management in contemporary organisations. Asia Pacific Journal of Human Resources, 55, 6-43. https://doi.org/10.1111/1744-7941.12111

Centers for Disease Control and Prevention. (2018). Disability and Health Data Systems (DHDS). Retrieved June 1, 2020, from https://www.cdc.gov/ncbddd/disabilityandhealth/dhds/index.html?CDC_AA_refVal=https\%3A\% 2F\%2Fwww.cdc.gov\%2Fncbddd\%2Fdisabilityandhealth\%2Fdhds.html

Chalofsky, N. (2003). An emerging construct for meaningful work. Human Resource Development International, 6(1), 69-83.

Cole, B.M., \& Salimath, M.S. (2012). Diversity identity management: An organizational perspective. Journal of Business Ethics, 116(1), 151-161. doi:10.1007/s10551-012-14664

Corbin, J., \& Strauss, A. (2015). Basics of qualitative research: Techniques and procedures for developing grounded theory (4th ed.). Newbury Park: Sage.

DiMaggio, P.J., \& Powell, W.W. (1983). The iron cage revisited: Institutional isomorphism and collective rationality in organizational fields. American Sociological Review, 48(2), 147-160.

Hindle, K., Gibson, B., \& David, A. (2010). Optimising employee ability in small firms: Employing people with a disability. Small Enterprise Research, 17(2), 207-212.

Kalargyrou, V. (2014). Gaining a competitive advantage with disability inclusion initiatives. Journal of Human Resources in Hospitality and Tourism, 13(2), 120-145.

Lindsay, S., Cagliostro, E., Albarico, M., Mortaji, N., \& Karon, L. (2018). A systematic review of the benefits of hiring people with disabilities. Journal of Occupational Rehabilitation, 28, 634-655. https://doi.org/10.1007/s10926-018-9756-z

Maxwell, J.A. (1996). Qualitative research design: An interactive approach. Thousand Oaks, CA: Sage Publications.

Miles, M.B., \& Huberman, A.M. (1994). Qualitative data analysis: An expanded sourcebook (2nd ed.). Thousand Oaks, CA: Sage Publications. 
Selznick, P. (1949). TVA and the Grass Roots. Berkeley: University of California Press.

U.S. Bureau of Labor Statistics. (2021). Persons with a Disability: Labor Force Characteristics - 2020.

Retrieved February 26, 2021, from https://www.bls.gov/news.release/pdf/disabl.pdf

Yeoman, R. (2014). Conceptualising meaningful work as a fundamental human need. Journal of Business Ethics, 125(2), 235-251. 